\title{
Article
}

\section{Severity and progression rate of cerebellar ataxia in 16q-linked autosomal dominant cerebellar ataxia (16q-ADCA) in the endemic Nagano area of Japan}

\author{
Kunihiro Yoshida, $\mathrm{MD}^{1}$, Yusaku Shimizu, $\mathrm{MD}^{2}$, Hiroshi Morita, $\mathrm{MD}^{1}$, \\ Tomomi Okano, $\mathrm{MD}^{3}$, Haruya Sakai, $\mathrm{PhD}^{4}$, Takako Ohata, $\mathrm{MD}^{4}$, \\ Naomichi Matsumoto, $\mathrm{MD}^{4}$, Katsuya Nakamura, $\mathrm{MD}^{1}$, Ko-ichi Tazawa, $\mathrm{MD}^{1}$, \\ Shinji Ohara, $\mathrm{MD}^{3}$, Kenichi Tabata, $\mathrm{MD}^{5}$, Atsushi Inoue, $\mathrm{MD}^{6}$, Shunichi Sato, $\mathrm{MD}^{7}$, \\ Yasuhiro Shimojima, $\mathrm{MD}^{8}$, Takeshi Hattori, $\mathrm{MD}^{8}$, Masao Ushiyama, $\mathrm{MD}^{9}$, \\ Shu-ichi Ikeda, $\mathrm{MD}^{1}$
}

\begin{abstract}
${ }^{1)}$ Department of Medicine (Neurology and Rheumatology), Shinshu University School of Medicine,
${ }^{2)}$ Department of Neurology, Ina General Hospital, ${ }^{3)}$ Department of Neurology, National

Chushin-Matsumoto Hospital, ${ }^{4}$ Department of Human Genetics, Yokohama City University Graduate School of Medicine, ${ }^{5}$ Department of Neurology, Saku General Hospital, ${ }^{6)}$ Department of Neurology, Nagano Prefectural Kiso Hospital, ${ }^{7)}$ Department of Neurology, Nagano Red Cross Hospital, ${ }^{8}$ Department of Neurology, Suwa Red Cross Hospital, ${ }^{9}$ Department of Neurology, Kenwakai Hospital
\end{abstract}

Address correspondence and reprint requests to Dr. Kunihiro Yoshida, Department of Medicine (Neurology and Rheumatology), Shinshu University School of Medicine, Asahi 3-1-1, Matsumoto 390-8621, Japan TEL; +81-263-37-2673, FAX; +81-263-37-3427 e-mail address; kyoshida@shinshu-u.ac.jp 
Disclosure: The authors report no conflicts of interest. 


\section{ABSTRACT}

16q22.1-linked autosomal dominant cerebellar ataxia (16q-ADCA) is a recently defined subtype of ADCA identified by a disease-specific C/T substitution in the 5'-UTR of the puratrophin-1 gene. In Nagano, the central mountainous district of the main island of Japan, 16q-ADCA and spinocerebellar ataxia type 6 (SCA6) are the most and second most prevalent subtypes of ADCA, respectively. Both subtypes are classified into Harding's ADCAIII, but little attention has been given to the differences in the severity and progression rate of cerebellar ataxia between 16q-ADCA and SCA6. We investigated the clinical severity and progression rate of cerebellar ataxia of 16q-ADCA patients using ICARS and SARA, and compared them with those of SCA6 patients. The age at onset was much higher in 16q-ADCA patients (60.1 \pm 9.8 years, $n=66)$ than in SCA6 patients ( $41.1 \pm 8.7$ years, $n=35)$. Clinical features of 16q-ADCA were basically consistent with pure cerebellar ataxia, as well as in SCA6, but gaze-evoked nystagmus was observed less frequently in 16q-ADCA patients than in SCA6 patients. When compared at almost the same disease duration after onset, the severity of cerebellar ataxia was a little higher, and the progression rate seemed more rapid in 16q-ADCA patients than in SCA6 patients, but the differences were not significant.

\section{GLOSSARY}

$\mathrm{ADCA}=$ autosomal dominant cerebellar ataxia, 16q-ADCA $=16 \mathrm{q} 22.1$-linked autosomal dominant cerebellar ataxia, $\mathrm{SCD}=$ spinocerebellar degeneration, SCA6 $=$ spinocerebellar ataxia type 6, ICARS = international cerebellar ataxia rating scales, SARA = scales for the assessment and rating of ataxia

\section{Key words}

cerebellar ataxia, 16q-ADCA, SCA6, ICARS, SARA, puratrophin-1 


\section{Introduction}

Autosomal dominant cerebellar ataxia (ADCA) is a genetically and clinically heterogeneous group of diseases characterized by a slowly progressive gait and truncal unsteadiness, dysarthria, and often associated with poor coordination of hands $[1,2]$. The incidence of the subtypes of ADCA is quite variable in countries [2]. Even in Japan, the incidence of the subtypes varies in different areas [3-5]. In general, SCA3/Machado-Joseph disease (MJD) or SCA6 are the most or second most predominant subtypes, and dentatorubral-pallidoluysian atrophy (DRPLA) is the third most prevalent subtype in Japan [2-5].

Recently, a new subtype called 16q22.1-linked ADCA (16q-ADCA) has become recognized [6-9]. A single nucleotide substitution $(-16 \mathrm{C}>\mathrm{T})$ in the 5' UTR of the gene encoding puratrophin-1 has been shown to be a disease-specific marker for 16q-ADCA [10, 11]. According to the availability of a diagnostic marker for 16q-ADCA, the prevalence of this subtype has been estimated as 8-17\% in ADCA families in Japan [12-16]. But, so far, this specific substitution has been found exclusively in the Japanese population

[17]. Thus, it is still unclear whether 16q-ADCA exists in countries other than Japan.

In our previous study, we have shown that 16q-ADCA is the most predominant subtype of ADCA in Nagano, which is located in the central, mountainous district of the main island of Japan [18, 19]. To date, we have analyzed 143 ADCA families from Nagano, and diagnosed 61 families (42.7\%) with 16q-ADCA and 29 (20.3\%) with SCA6. Thus, the frequency of 16q-ADCA in Nagano is much higher than in the other areas of Japan [12-16]. In particular, the frequency of 16q-ADCA is extremely high in the restricted areas of Nagano, 25/31 (80.6\%) in the southwest area (Ina-Kiso area), and 17/26 (65.4\%) in the east area (Saku area), reflecting the accumulation of 16q-ADCA families in these areas.

Clinically, both 16q-ADCA and SCA6 show pure cerebellar ataxia of late-onset [10-15, 19] and are classified into ADCA III according to Harding's criteria [20], but little attention has been given to the differences in the severity and progression rate of cerebellar ataxia between 16q-ADCA and SCA6 
patients. It is quite difficult to differentiate between 16q-ADCA and SCA6 without genetic testing. In fact, most families with 16q-ADCA in the Nagano area had been considered as having SCA6 before genetic testing.

In consideration of the high frequency of 16q-ADCA in Nagano and the clinical similarities of both subtypes, we have investigated the clinical features of 16q-ADCA in the endemic Nagano area in more detail. The main purpose of this study was to clarify if the clinical severity and progression rate of cerebellar ataxia were different between 16q-ADCA and SCA6. We also tried to verify whether or not the clinical features of 16q-ADCA patients in the endemic Nagano area were different from those in non-endemic areas in Japan. 


\section{Materials and Methods}

Subjects

To date, we have genetically confirmed 89 patients with 16q-ADCA from 61 families and 42 patients with SCA6 from 29 families in Nagano. From these families, we have recruited 66 patients with 16q-ADCA and 35 patients with SCA6 in this study. Some of the affected individuals with 16q-ADCA or SCA6, who were dead, institutionalized or no longer lived in Nagano, could not be examined by ourselves, but their medical records were available. This research protocol was approved independently by the Ethical Committee of Shinshu University School of Medicine and by the Committee for Ethical Issues at Yokohama City University Graduate School of Medicine.

Clinical evaluations

Detailed medical interviews and routine neurological examinations were done by expert neurologists. Age at onset was determined on the basis of the information provided by the patients or their close relatives. International cerebellar ataxia rating scales (ICARS) [21] and scales for the assessment and rating of ataxia (SARA) [22] were used for the assessment of cerebellar ataxia. The patients with 16q-ADCA $(n=52)$ or with SCA6 $(n=25)$ were evaluated with ICARS and SARA simultaneously by either of the first three authors. Hearing impairment was judged as positive when the patient felt difficulty in daily conversation. Audiograms were performed only for a minority of the patients.

Statistics

Statistical analysis was performed using the Student’s t-test (Microsoft Office Excel 2003). Regression analysis for ICARS and SARA data was done with Microsoft Office Excel 2003. The level of significance was set at $p<0.01$. 


\section{Results}

Neurological findings are summarized in Table 1. The age at onset was $60.1 \pm 9.8$ and $41.1 \pm 8.7$ in 16q-ADCA $(n=66)$ and SCA6 $(n=35)$, respectively. All the patients with 16q-ADCA showed gait unsteadiness with or without dysarthria as initial symptoms. Neither pyramidal nor extrapyramidal tract signs were common for 16q-ADCA, as well as for SCA6. Deep tendon reflexes were variable, but the Babinski reflex was generally negative in 16q-ADCA. Cognitive impairment was found in 4 patients (6.1\%) with 16q-ADCA. Hearing impairment was noted in 10 patients (15.2\%) with 16q-ADCA. All four patients with cognitive impairment were over 80 years (80-86 years, mean 81.8 years), and negative for other evident causes of dementia on brain MRI. Ten patients with hearing impairment were aged between 65-85 years (mean 78.9 years), and 3 of them had a history of repetitive otitis media. Three patients had both cognitive and hearing impairment. Mild or moderate hand tremor or head titubation were seen in some patients with 16q-ADCA, but only one patient underwent deep brain stimulation for disabling hand tremor.

The scores of ICARS and SARA are shown in Table 2, and the correlation between ICARS or SARA and age at examination or duration of illness is shown in Figure 1. The ICARS and SARA scores were well correlated in each patient. Both the total scores of ICARS and SARA were a little higher in 16q-ADCA patients than in SCA6 patients (Table 2), but the difference was not significant. Contrary to the other three subscores in ICARS, only the oculomotor disorders subscore was lower in 16q-ADCA patients $(1.8 \pm 1.4)$ than in SCA6 patients $(3.2 \pm 1.1)$ (Table 2). This is mainly because gaze-evoked nystagmus was more evident in SCA6 patients than in 16q-ADCA patients. The patients with persistent gaze-evoked nystagmus (gaze-evoked nystagmus score 2, or 3 on ICARS [21]) were observed more frequently in SCA6 $(17 / 25,68.0 \%)$ than in 16q-ADCA (6/52, 11.5\%). Cerebellar ataxia was slowly progressive in both 16q-ADCA and SCA6 patients, and the ICARS and SARA scores seemed to increase more rapidly in 16q-ADCA patients than in SCA6 patients (Figure 1), but the difference was not 
significant.

\section{Discussion}

16q-ADCA is the most prevalent subtype of ADCA (나.7\% in ADCA) in the Nagano area, where two main foci are identified in the southwest area (Ina-Kiso area) and east area (Saku area) of Nagano. In these areas, the incidence of spinocerebellar degeneration (SCD) is higher than in the other areas of Nagano, mainly because of an accumulation of 16q-ADCA families [19]. Having two large foci of 16q-ADCA, Nagano is considered to be an endemic area for 16q-ADCA in Japan.

In the present study, we first investigated the severity and progression rate of cerebellar ataxia in a large population of 16q-ADCA patients, using ICARS and SARA, and compared them with those in SCA6 patients. Both these scales have been shown to exhibit high inter-rater reliability [22, 23] and are widely used for evaluation for ataxic patients. Both the total scores of ICARS and SARA were a little higher in 16q-ADCA patients than in SCA6 patients at almost the same disease duration after onset. The rate of disease progression seemed more rapid in 16q-ADCA patients than in SCA6 patients. But the differences in the severity and progression rate were not significant. The major concern for interpreting the data is that the ages of the patients in both groups were considerably different, even though the mean disease duration was not different between the two groups. In particular, the mean age at onset of our SCA6 patients was younger than reported previously [24-27], although the reason for this was unknown. As a result, the mean age at examination in 16q-ADCA patients ( $72.5 \pm 8.5$ years, $n=52)$ was about 15 years older than in SCA6 patients (57.2 \pm 11.0 years, $n=25)$. Therefore, we should keep in mind that the effect of aging-related factors (e.g. degenerative arthropathy, disuse atrophy of lower extremities) might modify the clinical severity and progression rate more seriously for 16q-ADCA patients than for SCA6 patients.

Patients with 16q-ADCA in the endemic Nagano area showed pure cerebellar ataxia with late-onset, which is basically compatible with those in non-endemic areas in Japan (Table 3) [12-15]. The mean age 
at onset in our patients was almost the same as that in patients in non-endemic areas. This is in contrast to the case for transthyretin Met30-associated familial amyloid polyneuropathy (FAP TTR Met30). Nagano has been known to be one of worldwide foci for FAP TTR Met30. In FAP TTR Met30, patients from the endemic foci show features distinct from those of patients from non-endemic areas in Japan [28, 29]. For example, the age at onset is generally younger in the former than in the latter [28, 29]. A founder effect may be much more profound or disease modifying factors other than the causative gene mutation may be less influential in 16q-ADCA than in TTR FAP Met30.

As reported previously for 16q-ADCA patients in non-endemic areas [12-15], extracerebellar symptoms and signs were rare in our patients in the endemic Nagano area. In our study, cognitive and hearing impairment were observed in 4 (6.1\%) and 10 (15.2\%) patients, respectively. The mean ages of the patients with cognitive or hearing impairment were almost 80 years or over. This was supportive of the observation of Nozaki et al. [14] that extracerebellar features were observed more frequently in elderly patients aged over 65 years. Hayashi et al. reported that the frequency of dementia, pyramidal, exrapyramidal signs, and leukoaraiosis on brain MRI was considerable in their 12 patients with 16q-ADCA [16]. This observation was quite unique in terms of the high frequency of extracerebellar features and leukoaraiosis, when compared with reports from other districts of Japan including ours [12-15, 19]. In our series, leukoaraiosis was not seen in brain MRI, even in the 4 patients with cognitive impairment. The extracerebellar features and leukoaraiosis described by Hayashi et al [16] could be due to phenotypic variation of 16q-ADCA or a coincidental effect of other factors peculiar to their 16q-ADCA patients. Further clinical and neuropathological investigations are required to clarify the disease characteristics of 16q-ADCA.

Gaze-evoked nystagmus is one of the cardinal features of SCA6 [24-27]. This was again confirmed in our study. On the other hand, gaze-evoked nystagmus was less frequently observed in 16q-ADCA patients, reflecting that the oculomotor disorders subscores were significantly lower in 16q-ADCA patients than in 
SCA6 patients. The pathogenesis of gaze-evoked nystagmus has been discussed in association with the lesions pathologically involved [30]. Purkinje cell degeneration is the most prominent pathological finding in both SCA6 and 16q-ADCA [30, 31]. The difference in the frequency in gaze-evoked nystagmus between SCA6 and 16q-ADCA may be due to the difference of the degree and distribution of affected lesions between both diseases.

In conclusion, there was no clear difference in clinical features in 16q-ADCA patients from the endemic Nagano area and non-endemic areas in Japan. The mean age at onset in 16q-ADCA patients was significantly higher than that in SCA6 patients. The total scores of ICARS and SARA at almost the same disease duration were higher, but the oculomotor disorders subscore on ICRAS was lower in 16q-ADCA patients than in SCA6 patients. The progression rate of cerebellar ataxia judged by ICARS and SARA might be more rapid in 16q-ADCA patients than in SCA6 patients, but it is possible that other physical factors related to aging have a greater influence upon these scores in 16q-ADCA patients than in SCA6 patients.

\section{Acknowledgement}

We are grateful to the clinicians who provided us with information on their patients, and the patients and their families who participated in this study. This work was supported in part by a Grant-in-Aid for Science Research from the Ministry of Education, Science and Culture, Japan and a grant from the Research Committee for Ataxic Diseases, the Ministry of Health, Labor, and Welfare, Japan. 


\section{Figure legends}

Fig.1. Correlation between ICARS and age at examination (A) or duration of disease (B) in patients with 16q-ADCA $(n=52)$ and SCA6 $(n=25)$ is shown. Correlation between SARA and age at examination $(C)$ or duration of disease (D) in patients with 16q-ADCA $(n=52)$ and SCA6 $(n=25)$ is shown. Solid and dotted lines indicate linear regression lines for 16q-ADCA and SCA6, and closed and open circles indicate patients with 16q-ADCA and SCA6, respectively. 


\section{References}

1. Margolis RL. Dominant spinocerebellar ataxias: a molecular approach to classification, diagnosis, pathogenesis and the future. Expert Rev Mol Diagn 2003; 3: 715-32.

2. Schöls L, Bauer P, Schmidt T, Schulte T, Riess O. Autosomal dominant cerebellar ataxias: clinical features, genetics, and pathogenesis. Lancet Neurol 2004; 3: 291-304.

3. Takano H, Cancel G, Ikeuchi T, Lorenzetti D, Mawad R, Stevanin G, et al. Close associations between prevalences of dominantly inherited spinocerebellar ataxias with CAG-repeat expansions and frequencies of large normal CAG alleles in Japanese and Caucasian populations. Am J Hum Genet 1998; 63: 1060-6.

4. Maruyama H, Izumi Y, Morino H, Oda M, Toji H, Nakamura S, et al. Difference in disease-free survival curve and regional distribution according to subtype of spinocerebellar ataxia: a study of 1,286 Japanese patients. Am J Med Genet 2002; 114: 578-83.

5. Sasaki H, Yabe I, Tashiro K. The hereditary spinocerebellar ataxias in Japan. Cytogenet Genome Res 2003; 100: 198-205.

6. Nagaoka U, Takashima M, Ishikawa K, Yoshizawa K, Yoshizawa T, Ishikawa M, et al. A gene on SCA4 locus causes dominantly inherited pure cerebellar ataxia. Neurology 2000; 54: 1971-5.

7. Takashima M, Ishikawa K, Nagaoka U, Shoji S, Mizusawa H. A linkage disequilibrium at the candidate gene locus for 16q-linked autosomal dominant cerebellar ataxia type III in Japan. J Hum Genet 2001; 46: $167-71$.

8. Li M, Ishikawa K, Toru S, Tomimitsu H, Takashima M, Goto J, et al. Physical map and haplotype analysis of 16q-linked autosomal dominant cerebellar ataxia (ADCA) type III in Japan. J Hum Genet 
2003; 48: 111-8.

9. Hirano R, Takashima H, Okubo R, Tajima K, Okamoto Y, Ishida S, et al. Fine mapping of 16q-linked autosomal dominant cerebellar ataxia type III in Japanese families. Neurogenetics 2004; 5: 215-21.

10. Ishikawa K, Toru S, Tsunemi T, Li M, Kobayashi K, Yokota T, et al. An autosomal dominant cerebellar ataxia linked to chromosome $16 q 22.1$ is associated with a single-nucleotide substitution in the 5' untranslated region of the gene encoding a protein with spectrin repeat and rho guanine-nucleotide exchange-factor domains. Am J Hum Genet 2005; 77: 280-96.

11. Amino T, Ishikawa $\mathrm{K}$, Toru $\mathrm{S}$, Ishiguro $\mathrm{T}$, Sato $\mathrm{N}$, Tsunemi $\mathrm{T}$, et al. Redefining the disease locus of 16q22.1-linked autosomal dominant cerebellar ataxia. J Hum Genet 2007; 52: 643-9.

12. Ouyang Y, Sakoe K, Shimazaki H, Namekawa M, Ogawa T, Ando Y, et al. 16q-linked autosomal dominant cerebellar ataxia: a clinical and genetic study. J Neurol Sci 2006; 247: 180-6.

13. Onodera Y, Aoki M, Mizuno H, Warita H, Shiga Y, Itoyama Y. Clinical features of chromosome 16q22.1 linked autosomal dominant cerebellar ataxia in Japanese. Neurology 2006; 67: 1300-2. 14. Nozaki H, Ikeuchi T, Kawakami A, Kimura A, Koide R, Tsuchiya M, et al. Clinical and genetic characterizations of 16q-linked autosomal dominant spinocerebellar ataxia (AD-SCA) and frequency analysis of AD-SCA in the Japanese population. Mov Disord 2007; 22: 857-62.

15. Basri R, Yabe I, Soma H, Sasaki H. Spectrum and prevalence of autosomal dominant spinocerebellar ataxia in Hokkaido, the northern island of Japan: a study of 113 Japanese families. J Hum Genet 2007; 52: $848-55$.

16. Hayashi M, Adachi Y, Mori M, Nakano T, Nakashima K. Clinical and genetic epidemiological study of 16q22.1-linked autosomal dominant cerebellar ataxia in western Japan. Acta Neurol Scand 2007; 116: 
123-7.

17. Wieczorek S, Arning L, Alheite I, Epplen JT. Mutations of the puratrophin-1 (PLEKHG4) gene on chromosome 16q22.1 are not a common genetic cause of cerebellar ataxia in a European population. J Hum Genet 2006; 51: 363-7.

18. Shimizu Y, Yoshida K, Okano T, Ohara S, Hashimoto T, Fukushima Y, et al. Regional features of autosomal-dominant cerebellar ataxia in Nagano: Clinical and molecular genetic analysis of 86 families. J Hum Genet 2004; 49: 610-6.

19. Ohata T, Yoshida K, Sakai H, Hamanoue H, Mizuguchi T, Shimizu Y, et al. A -16C>T substitution in the 5' UTR of the puratrophin-1 gene is prevalent in autosomal dominant cerebellar ataxia in Nagano. J Hum Genet 2006; 51: 461-6.

20. Harding AE. Clinical features and classification of inherited ataxias. Adv Neurol 1993; 61: 1-14.

21. Trouillas P, Takayanagi T, Hallett M, Currier RD, Subramony SH, Wessel K, et al. International Cooperative Ataxia Rating Scale for pharmacological assessment of the cerebellar syndrome. The Ataxia Neuropharmacology Committee of the World Federation of Neurology. J Neurol Sci 1997; 145: 205-11.

22. Schmitz-Hübsch T, du Montcel ST, Baliko L, Berciano J, Boesch S, Depondt C, et al. Scale for the assessment and rating of ataxia: development of a new clinical scale. Neurology 2006; 66: 1717-20.

23. Storey E, Tuck K, Hester R, Hughes A, Churchyard A. Inter-rater reliability of the international cooperative ataxia rating scale (ICARS). Mov Disord 2004; 19: 190-212.

24. Riess O, Schöls L, Böttger H, Nolte D, Vieira-Saecker, AMM, Schimming C, et al. SCA6 is caused by moderate CAG expansion in the $\alpha_{1 \mathrm{~A}}$-voltage-dependent calcium channel gene. Hum Molec Genet 1997; 6: 1289-93 
25. Ikeuchi T, Takano H, Koide R, Horikawa Y, Honma Y, Onishi Y, et al. Spinocerebellar ataxia type 6: CAG repeat expansion in $\alpha_{1 \mathrm{~A}}$ voltage-dependent calcium channel gene and clinical variations in Japanese population. Ann Neurol 1997; 42: 879-84.

26. Matsumura R, Futamura N, Fujimoto Y, Yanagimoto S, Horikawa H, Suzumura A, et al. Spinocerebellar ataxia type 6: molecular and clinical features of 35 Japanese patients including one homozygous for the CAG repeat expansion. Neurology 1997; 49: 1238-43.

27. Takahashi H, Ishikawa K, Tsutsumi T, Fujigasaki H, Kawata A, Okiyama R, et al. A clinical and genetic study in a large cohort of patients with spinocerebellar ataxia type 6. J Hum Genet 2004; 49: 256-64.

28. Misu K, Hattori N, Nagamatsu M, Ikeda S, Ando Y, Nakazato M, et al. Late-onset familial amyloid polyneuropathy type I (transthyretin Met30-associated familial amyloid polyneuropathy) unrelated to endemic focus in Japan. clinicopathological and genetic features. Brain 1999; 122: 1951-62.

29. Koike H, Misu K, Ikeda S, Ando Y, Nakazato M, Ando E, et al. Type I (transthyretin Met30) familial amyloid polyneuropathy in Japan. early- vs late-onset form. Arch Neurol 2002; 59: 1771-6.

30. Gomez CM, Thompson RM, Gammack JT, Perlman SL, Dobyns WB, Truwit CL, et al. Spinocerebellar ataxia type 6: gaze-evoked and vertical nystagmus, Purkinje cell degeneration, and variable age of onset. Ann Neurol 1997; 42: 933-50.

31. Owada K, Ishikawa K, Toru S, Ishida G, Gomyoda M, Tao O, et al. A clinical, genetic, and neuropathologic study in a family with 16q-linked ADCA type III. Neurology 2005; 65: 629-32. 
Table 1 Clinical characteristics of 16q-ADCA and SCA6

\begin{tabular}{|c|c|c|}
\hline & $16 \mathrm{q}-\operatorname{ADCA}(\mathrm{n}=66)$ & SCA6 $(n=35)$ \\
\hline male:female & $34: 32$ & $19: 16$ \\
\hline age at onset (years) & $60.1 \pm 9.8$ (range $30-78$ ) & $41.1 \pm 8.7{\text { (range } 28-60)^{*}}^{*}$ \\
\hline age at examination (years) & $72.6 \pm 8.9$ (range 54-86) & $55.4 \pm 10.9$ (range $36-75)^{*}$ \\
\hline duration of disease (years) & $12.3 \pm 8.1$ & $14.9 \pm 9.1$ \\
\hline \multicolumn{3}{|l|}{ clinical characteristics (\%) } \\
\hline cognitive impairment & $4(6.1)$ & $0(0)$ \\
\hline \multicolumn{3}{|l|}{ deep tendon reflex } \\
\hline normal & $43(65.2)$ & $24(68.6)$ \\
\hline increased & $11(16.7)$ & $5(14.3)$ \\
\hline decreased-lost & $12(18.2)$ & $6(17.1)$ \\
\hline positive Babinski reflex & $2(3.0)$ & $0(0)$ \\
\hline parkinsonism & $0(0)$ & $0(0)$ \\
\hline hearing impairment & $10(15.2)$ & $1(2.9)$ \\
\hline
\end{tabular}

${ }^{*} p<0.01$ 
Table 2 ICARS and SARA scores for 16q-ADCA and SCA6

$\begin{array}{ll}16 \mathrm{q}-\mathrm{ADCA} & \text { SCA6 } \\ (\mathrm{n}=52) & (\mathrm{n}=25)\end{array}$

age at onset (years)

$59.2 \pm 9.6 \quad 42.0 \pm 8.3^{*}$

(range 30-78) (range 32-58)

age at examination (years)

$72.5 \pm 8.5 \quad 57.2 \pm 11.0^{*}$

duration of disease (years)

$13.3 \pm 8.9 \quad 15.2 \pm 9.5$

ICARS score (total/100)

$37.3 \pm 16.8 \quad 35.1 \pm 16.0$

I. posture and gait disturbance subscore/52

$15.8 \pm 9.3$

$14.2 \pm 7.5$

II. kinetic functions subscore/34

$16.6 \pm 7.4 \quad 14.6 \pm 8.1$

III. speech disorders subscore/8

$3.2 \pm 1.4$

$3.0 \pm 1.1$

IV. oculomotor disorders subscore/6

$1.8 \pm 1.4$

$3.2 \pm 1.1^{*}$

SARA score (total/40)

$14.9 \pm 6.9$

$13.0 \pm 6.4$

${ }^{*} p<0.01$ 
Table 3 Clinical summary of Japanese patients with 16q-ADCA

\begin{tabular}{|c|c|c|c|c|c|c|}
\hline & $\begin{array}{l}\text { Ouyang et al. }{ }^{12} \\
(\mathrm{n}=22)\end{array}$ & $\begin{array}{l}\text { Onodera et al. }{ }^{13} \\
(\mathrm{n}=32)\end{array}$ & $\begin{array}{l}\text { Nozaki et al. }{ }^{14} \\
(\mathrm{n}=65)\end{array}$ & $\begin{array}{l}\text { Basri et al. }{ }^{15} \\
(\mathrm{n}=10)\end{array}$ & $\begin{array}{l}\text { Hayashi et al. }{ }^{16} \\
(\mathrm{n}=12)\end{array}$ & $\begin{array}{l}\text { this study } \\
(\mathrm{n}=66)\end{array}$ \\
\hline Age at onset (years) & 61.8 & $57.7 \pm 7.1$ & $59.1 \pm 6.9$ & 58.0 & $62.8 \pm 7.9$ & $60.1 \pm 9.8$ \\
\hline Age at examination (years) & 74.5 & nd & $67.3 \pm 8.4$ & 66.9 & nd & $72.6 \pm 8.9$ \\
\hline Disease duration (years) & 12.5 & $8.4 \pm 6.8$ & $8.2 \pm 6.1$ & 9.1 & nd & $12.3 \pm 8.1$ \\
\hline Clinical symptoms and signs & & & & & & \\
\hline $\begin{array}{l}\text { Dementia } \\
\text { Deep tendon reflexes }\end{array}$ & nd & 0 & 5 & 0 & 58 & 6.1 \\
\hline increased & 54.5 & 0 & 33 & 0 & $(58)^{*}$ & 16.7 \\
\hline decreased or lost & 13.6 & nd & 11 & 10 & nd & 18.2 \\
\hline Positive Babinski reflex & 0 & 0 & nd & 0 & $(58)^{*}$ & 3.0 \\
\hline Extrapyramidal disorders & nd & 0 & $8^{\#}$ & $0^{\S}$ & 50 & 0 \\
\hline Hearing impairment & 37.5 & 3 & nd & 20 & nd & 15.2 \\
\hline
\end{tabular}

nd; not described, ף; dystonia or rigidity, \#; involuntary movement, §; dystonia, *; pyramidal signs (it is unclear what the authors indicated exactly by “pyramidal signs” [16].) 


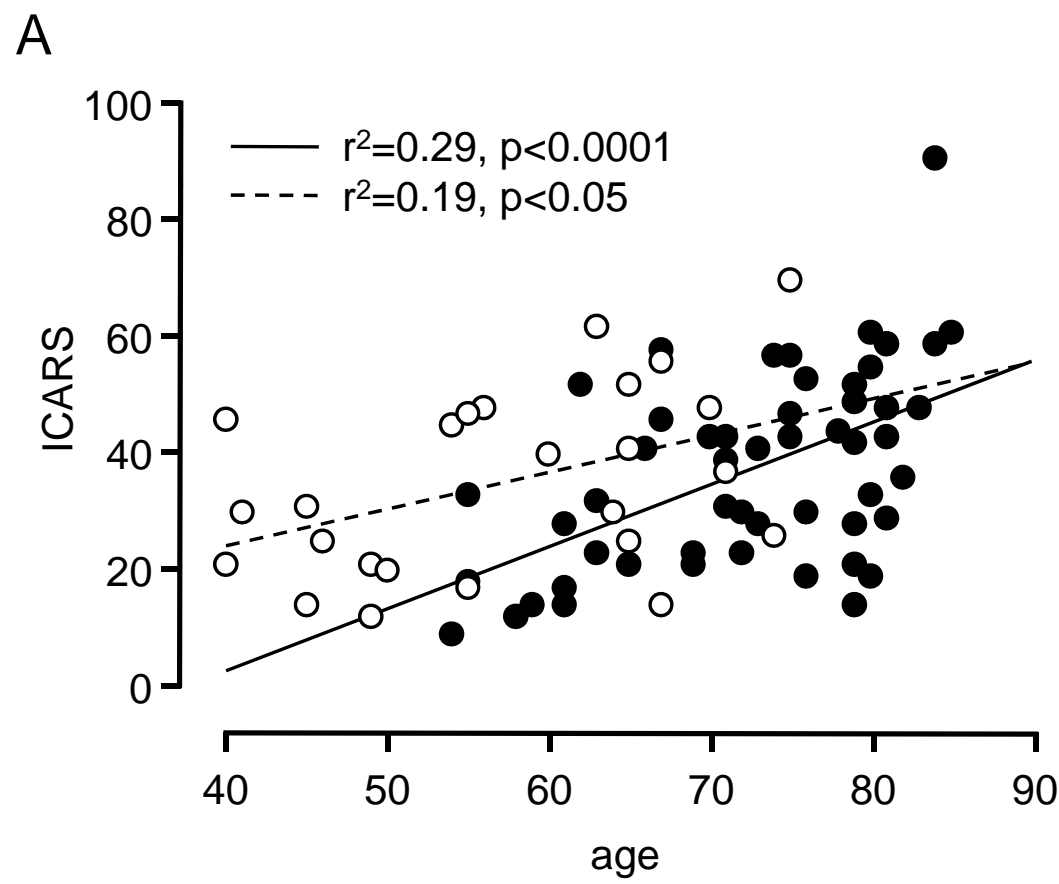

B
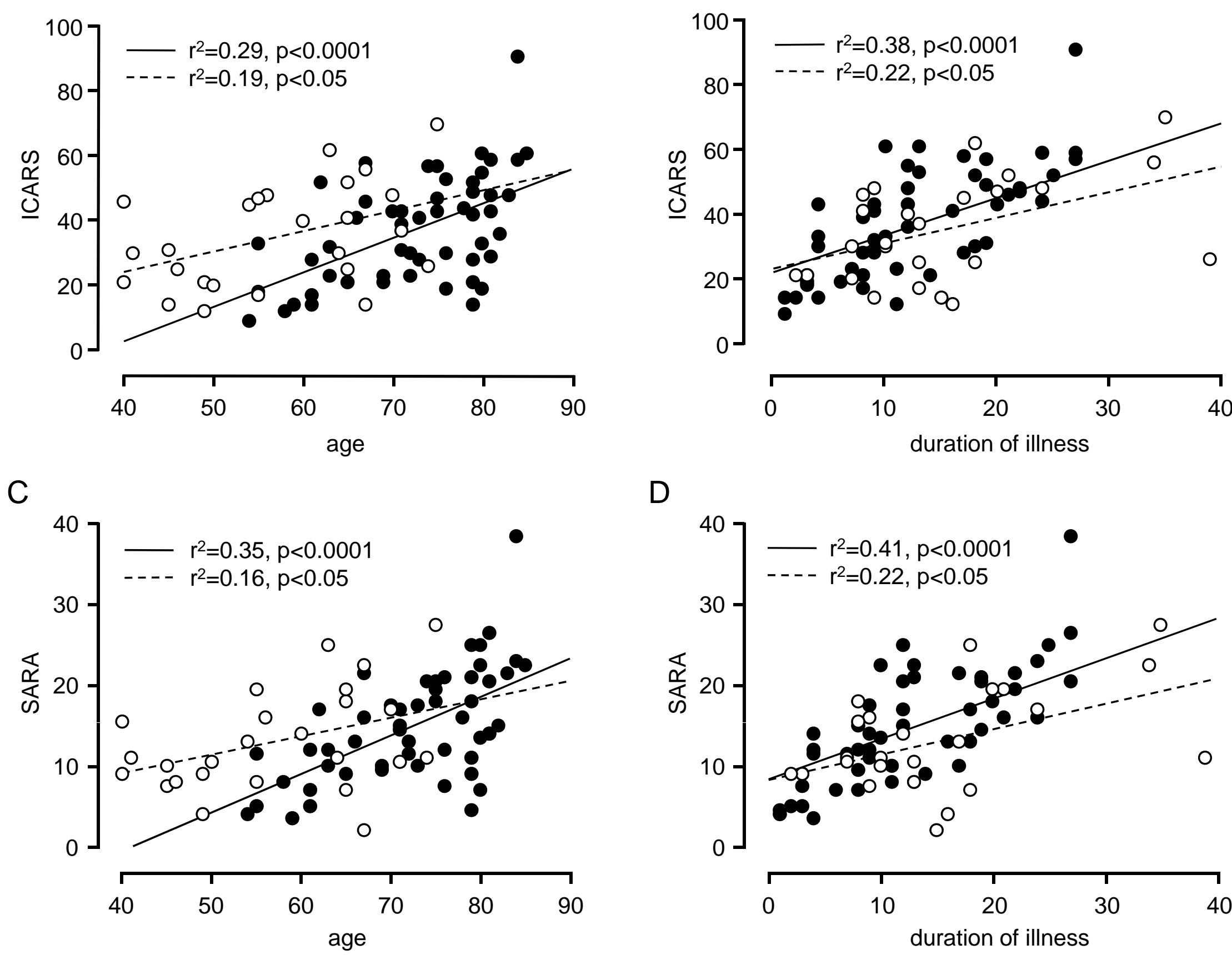

D

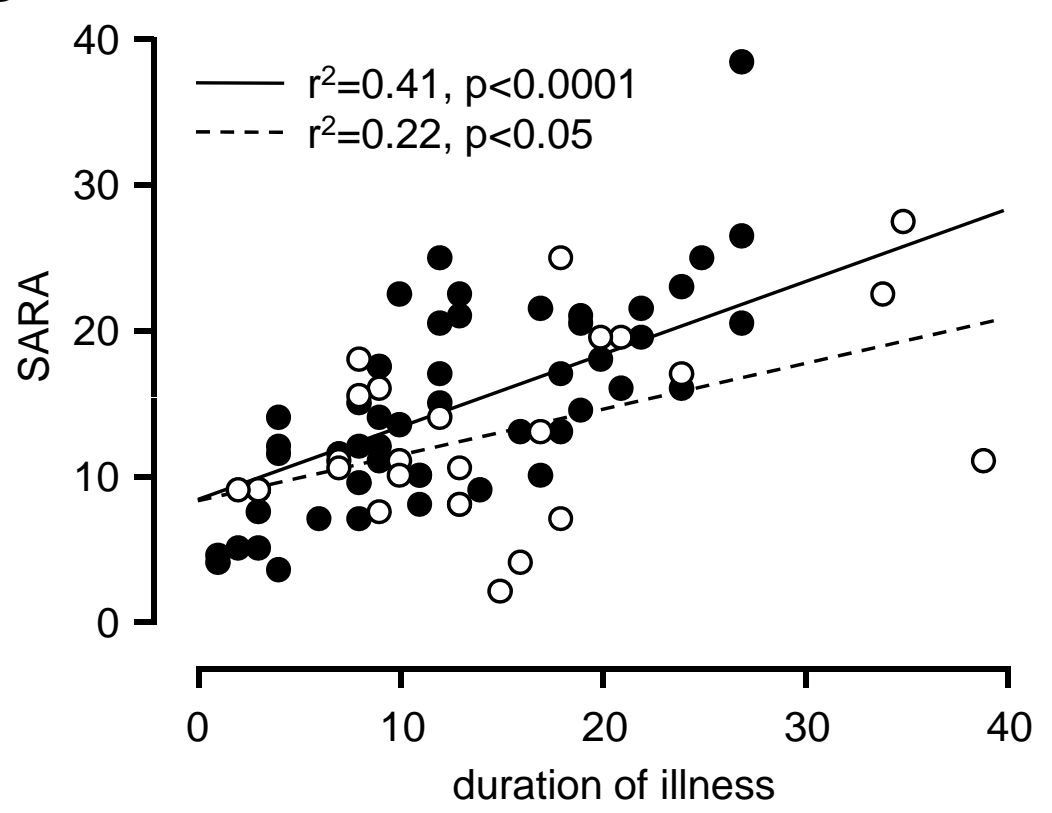

